\title{
ARRAW: Anti-Resonant Reflecting Acoustic Waveguide for efficient Brillouin scattering
}

\author{
M. K. Schmidt*a, M. C. O'Brien ${ }^{\text {a,b }}$, C. G. Poulton ${ }^{\mathrm{c}}$, M. J. Steel ${ }^{\mathrm{a}}$ \\ ${ }^{a}$ Macquarie Centre for Quantum Engineering (MQCQE), MQ Photonics Research Centre \\ Department of Physics and Astronomy, Macquarie University, NSW 2109, Australia. \\ ${ }^{b}$ School of Physics, The University of New South Wales, NSW 2052, Australia. \\ ${ }^{c}$ School of Mathematical and Physical Sciences, University of Technology Sydney, NSW 2007, Australia. \\ mikolaj.schmidt@mq.edu.au
}

\begin{abstract}
We introduce Anti-Resonant Reflecting Acoustic Waveguides (ARRAWs), implementing guidance of $\mathrm{GHz}$ acoustics waves through anti-resonant reflection in the cladding layer. We show that ARRAWs can simultaneously guide mid-IR optical modes, and support strong Brillouin Scattering. (C) 2020 The Author(s)
\end{abstract}

\section{Introduction}

Almost all conventional optical step-index waveguides are unsuitable for supporting low-loss propagation of $\mathrm{GHz}$ acoustic waves. This is because the high refractive index materials making up the core of optical waveguides tend to support acoustic waves propagating at larger velocities than in the low-refractive index cladding layers. Consequently, acoustic waves do not experience total internal reflection (TIR) at the corecladding interface, and freely dissipate into the cladding. Conversely, acoustic waveguides relying on a reversed design - with the acoustically slow material making up the core, and the fast material the cladding - usually do not guide optical waves, due to a general association between refractive index and material density. Therefore, if we are to pursue systems implementing efficient Brillouin interaction between propagating optical and acoustic waves [1], we need to look beyond the simple physics of TIR.

\section{Acoustic anti-resonant guidance}

In this work, we propose a novel class of waveguides capable of supporting the simultaneous propagation of co-localized optical and acoustic waves, based on the concept of Anti-Resonant Reflection Optical Waveguides (ARROWs) [2]. The acoustic analogues of such waveguides - Anti-Resonant Reflecting Acoustic Waveguides (ARRAWs), are capable of guiding acoustic waves through an acoustically fast core due to anti-resonances in the acoustically slow cladding (see schematic of a silicon/silica/silicon cylindrical waveguide in Fig. 1(a)). Furthermore, such ARRAWs can simultaneously support the conventional TIR guidance of light in the high refractive index core, and consequently amplify optoacoustic interactions between the co-localized optical and acoustic waves.
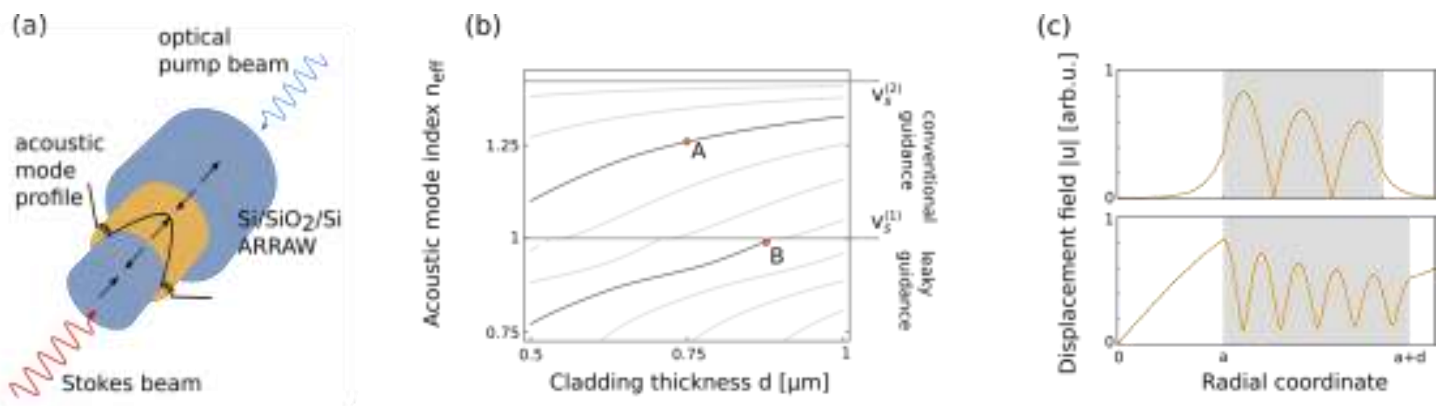

Fig. 1. (a) Schematic of the Anti-Resonant Reflecting Acoustic Waveguide (ARRAW) with silicon/silica/silicon structure supporting longitudinal anti-resonant modes and backwards SBS. By carefully choosing the thickness of the silica layer we can induce the simultaneous conventional optical guidance (through Total Internal Reflection), and anti-resonant guidance of acoustic waves in the core.

ARRAW modes are identified as lossy modes with phase velocities immediately above the fastest relevant sound line of the waveguide materials (for purely torsional modes this is the fastest shear wave velocity in bulk; for modes with shear and longitudinal components, this is the fastest - typically, longitudinal - wave velocity in bulk), at the local minimum of propagation losses. The lossy character ensures that the modes carry energy through all the layers of the structure, while the minimum suggests that in one of those layers modes exhibit an anti-resonant reflection.

In Fig. 1(b) we show the effective acoustic mode index of pure torsional modes a cylindrical waveguide with silicon core and outer cladding, and silica inner cladding layer, for increasing inner cladding (anti-resonant layer) thickness. Acoustic frequency is set to $2 \pi 15 \mathrm{GHz}$, core radius - to $0.5 \mu \mathrm{m}$, and silicon and silica are 
treated as isotropic materials with densities 2329 and $2203 \mathrm{~kg} / \mathrm{m}^{3}$, Poisson ratios 0.22 and 0.17 , and Young moduli of 162 and $73.1 \mathrm{GPa}$, respectively. Modes with effective index between 1 and 1.42 (ratio of shear velocities in silicon and silica), for example mode A, are conventionally guided in the inner cladding layer - see displacement field distribution along the radial direction in Fig. 1(c). Conversely, modes with effective index below 1 are lossy - in that region, just below the shear velocity line (denoted by $n_{\text {eff }}=1$ ), we find ARRAW mode B with distribution of displacement field as shown in Fig. 1(d).

\section{Suppressing losses and enabling Brillouin scattering in ARRAWs}

ARRAW modes discussed in Fig. 1 do not provide a significant confinement of the acoustic field to the core mainly because the core of these waveguides is too small. Now we show that ARRAWs can be optimized for application in backwards Brillouin scattering by considering structures with a larger core.

In Fig. 2(a) we show a section of the dispersion relation calculated for dilatational modes (with shear and longitudinal components) of a silicon/silica/silicon cylindrical waveguide, same as discussed in Fig. 1, but with the larger core of $2 \mu \mathrm{m}$. Sections of the dispersion marked with blue lines correspond to ARRAW modes characterized with quality factors (denoted in Fig. 2(a) as orange dashed lines) $Q_{m}>100$, and are found right below the fastest sound line describing longitudinal waves in silicon. In Fig. 2(b) we show that behavior by analyzing in detail mode $\mathrm{C}$ marked with a red dot in the plots of effective mode index and quality factor. We plot the radial distribution of the displacement field associated with that mode (green line), finding a significant confinement of the mode to the core, and a high order anti-resonance in the inner cladding layer. This mode can be used to mediate backwards Brillouin scattering between two conventionally guided optical modes $\mathrm{HE}_{17}$, characterized with vacuum wavelength of $1.92 \mu \mathrm{m}$, and axial component of the Poynting vector shown with dashed red line. With both modes overlapping in the core of the structure, and quality factor of mechanical mode approaching 2000, Brillouin gain - dominated by the photoelasticity in the silicon core - is calculated as $90(\mathrm{Wm})^{-1}$.

(a)

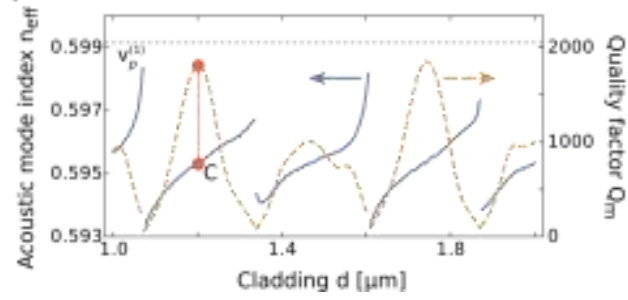

(b)

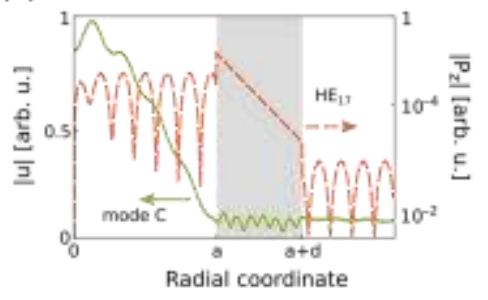

Fig. 2. Brillouin interaction between conventionally guided optical and acoustic ARRAW modes of a layered cylindrical waveguide. (a) Dispersion relation (blue lines) and mechanical quality factors $\mathrm{Q}_{\mathrm{m}}$ (dashed orange lines) of the highest- $\mathrm{Q}_{\mathrm{m}}$ ARRAW modes for increasing cladding thickness $\mathrm{d}$ for the cylindrical structure with core radius a $=2 \mu \mathrm{m}$ and operating at frequency $2 \pi 16 \mathrm{GHz}$. (b) Displacement field $|\mathrm{u}|$ of ARRAW mode $\mathrm{C}$ (green solid lines), and the axial Poynting vector $\left|\mathrm{P}_{\mathrm{z}}\right|$ of the optical mode $\mathrm{HE}_{17}$ (dashed red line).

While larger Brillouin gain can be achieved through increasing the acoustic frequency of the modes, or shrinking the geometric dimensions of the waveguide, the advantage of our approach lies in the relative simplicity of the design, and the choice of materials - silicon and silica. Achieving similar Brillouin gain typically requires extensive engineering of the acoustic dissipation through suspending the waveguide $[1,4]$ or relying on high refractive index, soft materials, such as chalcogenides [3].

\section{Summary and outlook}

Our results demonstrate the feasibility of a new type of SBS waveguides. The nonlinear optical response of these systems can be tuned across an entire near-IR and mid-IR range, and implemented in hybrid approaches in more complex geometries, for example to suppress the acoustic dissipation into the substrate in rib waveguides.

\section{References}

[1] B.J. Eggleton, C.G. Poulton, P.T. Rakich, M.J. Steel, and G. Bahl, “Brillouin integrated photonics,” Nature Phot., 13, 664 (2019).

[2] N.M. Litchinitser, S.C. Dunn, B. Usner, B.J. Eggleton, T.P. White, R.C. McPhedran, and C.M. de Sterke, "Resonances in microstructured optical waveguides," Opt. Express 11, 1243 (2003).

[3] R. Pant, C.G. Poulton, D.-Y. Choi, H. Mcfarlane, S. Hile, E. Li, L. Thevenaz, B. Luther-Davies, S.J. Madden, and B.J. Eggleton, “Onchip stimulated Brillouin scattering," Opt. Express 19, 8285 (2011).

[4] M.K. Schmidt, C.G. Poulton, G.Z. Mashanovich, G.T. Reed, B.J. Eggleton, and M.J. Steel., " Suspended mid-infrared waveguides for Stimulated Brillouin Scattering," Opt. Express 27, 4976 (2019).

[5] M.K. Schmidt, M.C. O’Brien, M.J. Steel, and C.G. Poulton, "ARRAW: Anti-Resonant Reflecting Acoustic Waveguides," arXiv preprint, arXiv:1909.01632 (2020); in press. 